\title{
Strategies of Collateral Blood Flow Assessment in Ischemic Stroke: Prediction of the Follow-Up Infarct Volume in Conventional and Dynamic CTA
}

S.E. Beyer, K.M. Thierfelder, L. von Baumgarten, M. Rottenkolber, F.G. Meinel, H. Janssen, B. Ertl-Wagner, M.F. Reiser, and W.H. Sommer

\begin{abstract}
BACKGROUND AND PURPOSE: Collateral blood flow is an important prognostic marker in the acute stroke situation but approaches for assessment vary widely. Our aim was to compare strategies of collateral blood flow assessment in dynamic and conventional CTA in their ability to predict the follow-up infarction volume.
\end{abstract}

MATERIALS AND METHODS: We retrospectively included all patients with an M1 occlusion from an existing cohort of 1912 consecutive patients who underwent initial multimodal stroke CT and follow-up MR imaging or nonenhanced CT. Collateralization was assessed in both conventional CT angiography and dynamic CT angiography by using 3 different collateral grading scores and segmentation of the volume of hypoattenuation. Arterial, arteriovenous, and venous phases were reconstructed for dynamic CT angiography, and all collateral scores and the volume of hypoattenuation were individually assessed for all phases. Different grading systems were compared by using the Bayesian information criterion calculated for multivariate regression analyses (Bayesian information criterion difference = 2-6, "positive"; Bayesian information criterion difference $=6-10$, "strong"; Bayesian information criterion difference $=>10$, "very strong").

RESULTS: One hundred thirty-six patients (mean age, 70.4 years; male sex, 41.2\%) were included. In the multivariate analysis, models containing the volume of hypoattenuation showed a significantly better model fit than models containing any of the 3 collateral grading scores in conventional CT angiography (Bayesian information criterion difference $=>10$ ) and dynamic CT angiography (Bayesian information criterion difference $=>10$ ). All grading systems showed the best model fit in the arteriovenous phase. For the volume of hypoattenuation, model fit was significantly higher for models containing the volume of hypoattenuation as assessed in the arteriovenous phase of dynamic CT angiography compared with the venous phase (Bayesian information criterion difference $=6.2$ ) and the arterial phase of dynamic CT angiography (Bayesian information criterion difference $=>10$ ) and in comparison with conventional CT angiography (Bayesian information criterion difference $=>10$ ).

CONCLUSIONS: The use of dynamic CT angiography within the arteriovenous phase by using quantification of the volume of hypoattenuation is the superior technique for assessment of collateralization among the tested approaches.

ABBREVIATIONS: $\mathrm{BIC}=$ Bayesian information criterion; $\mathrm{NECT}=$ nonenhanced $\mathrm{CT} ; \mathrm{VH}=$ volume of hypoattenuation

n acute ischemic stroke, leptomeningeal collateral vessels provide blood flow to the ischemic bed of the occluded artery. ${ }^{1,2}$ The extent of collateralization is an independent predictor of a small-lesion volume on follow-up imaging ${ }^{3}$ and a favorable clinical outcome. ${ }^{4}$ It has been further shown to predict response to

Received June 14, 2014; accepted after revision August 7.

From the Institute for Clinical Radiology (S.E.B., K.M.T., F.G.M., B.E.-W., M.F.R., W.H.S.), Departments of Neurology (L.v.B.) and Neuroradiology (H.J.), Ludwig Maximilians University Hospital Munich, Munich, Germany; and Department of Medical Informatics, Biometry and Epidemiology (M.R.), Ludwig Maximilians University Munich, Munich, Germany.

S.E. Beyer and K.M. Thierfelder contributed equally to this work.

Please address correspondence to Wieland H. Sommer, MD, MPH, Institute for Clinical Radiology, Ludwig Maximilians University Hospital Munich, Marchioninistr 15, 81377 Munich, Germany; e-mail: Wieland.Sommer@med.uni-muenchen.de intravenous thrombolysis ${ }^{5}$ and to determine penumbral tissue loss. ${ }^{6}$ Noninvasive imaging of collaterals can be performed by using CT angiography ${ }^{7,8}$ or MR angiography. ${ }^{9}$ CTA offers the advantages of a wide availability and short scanning times.

One strategy to assess collateral blood flow in CTA is through grading of collateral vessels in the territory of the occluded artery. ${ }^{4,7,8}$ Currently, several different grading scores have been published, ${ }^{10}$ which differ not only in the number of categories of each score but also in the type of assessment (ie, an assessment relative to the contralateral hemisphere or absolute quantification). The 3 most commonly used scores in the literature include

Indicates article with supplemental on-line tables.

Indicates article with supplemental on-line photo.

http://dx.doi.org/10.3174/ajnr.A4131 
the following: 1) an absolute score assessing the percentage of the ischemic bed in which collateral filling is seen, ${ }^{3}$ ) a relative score comparing collateral vessel enhancement within the ischemic bed with similar contralateral vessels, ${ }^{8}$ and 3 ) a detailed relative score comparing collateral vessel enhancement with similar contralateral vessels within each ASPECTS region. ${ }^{11}$

Another strategy to assess collateral blood flow is through quantification of hypoattenuated brain tissue in CTA source images. ${ }^{12-15}$ Whereas hypoattenuation in nonenhanced CT (NECT) images represents the brain tissue net water uptake, ${ }^{16}$ hypoattenuation in CT angiography suggests an area receiving limited blood flow, which has been shown to correlate with initial diffusion-weighted images ${ }^{15}$ and follow-up lesion size. ${ }^{14}$ However, this correlation might be heavily influenced by the time point of image acquisition. ${ }^{13}$

Recently, Smit et al $^{17}$ demonstrated that conventional CTA can fail to capture delayed collateral enhancement. Delay-insensitive CT angiography with acquisition of multiple timeframes can help overcome this limitation. Its value in assessing the maximal extent of collateralization has been shown in several studies. ${ }^{5,17,18}$ However, the time point of image analysis might play an important role in correctly assessing the prognostic value of the collateralization.

The aim of our study was the following: 1) to determine the optimal time-phase for reconstruction of the dynamic CTA images to best predict follow-up lesion volume, and 2) to compare the predictive value of the volume of hypoattenuation with different collateral vessel grading scores.

\section{MATERIALS AND METHODS Study Design and Population}

The institutional review board approved the retrospective study and waived the requirement for informed consent. Our initial cohort consisted of 1912 consecutive patients who underwent initial whole-brain CT perfusion for suspected acute ischemic stroke at our institution between April 2009 and April 2014. From this cohort, we included all patients with complete occlusion of the middle cerebral artery in the M1 segment, with or without an occlusion of the internal carotid artery, on both conventional and dynamic CTA. We excluded patients with the following:

1) Incomplete or missing whole-brain CTP raw datasets

2) Missing follow-up NECT or MR imaging acquired at least 1 day after initial CT perfusion imaging ${ }^{19}$

3) Nondiagnostic quality of conventional CTA or dynamic CTA.

Data on the time of symptom onset, treatment with intravenous thrombolysis, and mechanical recanalization were collected by review of the medical charts.

\section{CT Examination Protocol}

The multiparametric CT protocol consisted of an NECT to exclude intracranial hemorrhage, supra-aortic CTA, and wholebrain CT perfusion, all performed by using one of the following CT scanners: Somatom Definition AS+, a 128-section CT scanner; Somatom Definition Flash, a 128-section dual-source CT scanner; and Somatom Definition Edge, a 128-section CT scanner (all by Siemens, Erlangen, Germany).

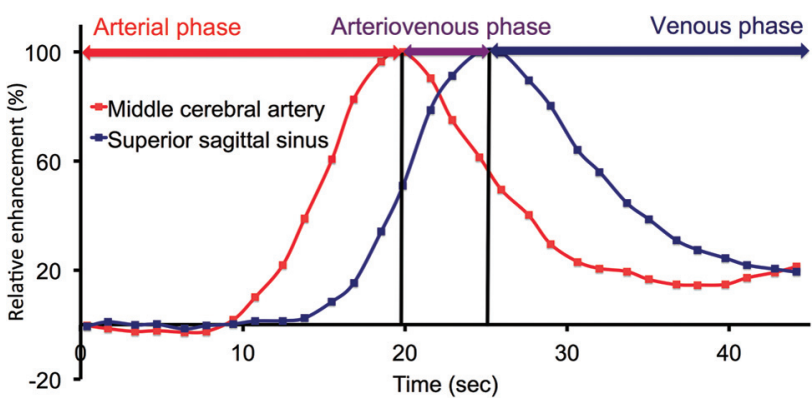

FIG 1. Definition of arterial, arteriovenous, and venous phases. Example of the separation the 3 phases by using the time-to-peak contrast enhancement of the contralateral middle cerebral artery and the superior sagittal sinus in a typical patient.

Conventional CT angiography was acquired in a caudocranial direction with a 2 -second delay after bolus triggering in the aortic arch (cutoff value $=100 \mathrm{HU}$ ). Images of whole-brain CTP were obtained with 0.6 -mm collimation and $100-\mathrm{mm}$ scan coverage in the $\mathrm{z}$-axis by using a toggling-table technique. One scan was acquired every 1.5 seconds. Tube voltage and current were $80 \mathrm{kV}$ and $200 \mathrm{mAs}$, respectively. CT dose index was $276 \mathrm{mGy}$. A total of $35 \mathrm{~mL}$ of iodinated contrast agent was administered at a flow rate of $5 \mathrm{~mL} / \mathrm{s}$, followed by a saline flush of $40 \mathrm{~mL}$ at $5 \mathrm{~mL} / \mathrm{s}$. Thirtyone axial sections were reconstructed per view with a thickness of $10 \mathrm{~mm}$ and an increment of $3 \mathrm{~mm}$.

\section{Dynamic CT Angiography Image Processing}

$\mathrm{CT}$ perfusion raw datasets were reconstructed as dynamic angiographies by using the syngo CT Dynamic Angio module and syngo via, VA 20 (Siemens). The image processing included motion correction and automated bone removal. Dynamic angiographies were then represented as source images and temporal MIPs. Computation of temporal MIPs included a 4D noise reduction; our approach was previously described by Smit et al. ${ }^{20}$ We created 3 different temporal MIPs representing the arterial phase, the arteriovenous phase, and the venous phase, to determine differences in collateral vessel enhancement with time. We created temporal MIPs of the different phases rather than using source images acquired at a single time point, to optimize quality because temporal MIP computation included the aforementioned 4D noise reduction. This process has been shown to increase the signal-tonoise ratio, vessel contour, detail visibility, and overall image quality. ${ }^{20}$ For standardization and adjustment for interindividual differences in circulation times and bolus dispersion, we used the time point of peak contrast enhancement of the contralateral MCA to separate arterial and arteriovenous phases and the time point of peak contrast enhancement of the superior sagittal sinus to separate arteriovenous and venous phases (Fig 1).

\section{Image Analysis of CTA and Dynamic CTA}

Quantification of Regions of Hypoattenuation. OsiriX, Version 4.0, imaging software (http:// www.osirix-viewer.com) was used for $3 \mathrm{D}$ assessment of regions of hypoattenuation by using a method previously described for CT perfusion deficit assessment. ${ }^{21}$ We manually segmented the region of hypoattenuation on every axial section of the temporal MIPs by using the OsiriX Closed Polygon Tool to create a region of interest; thus, the extent 

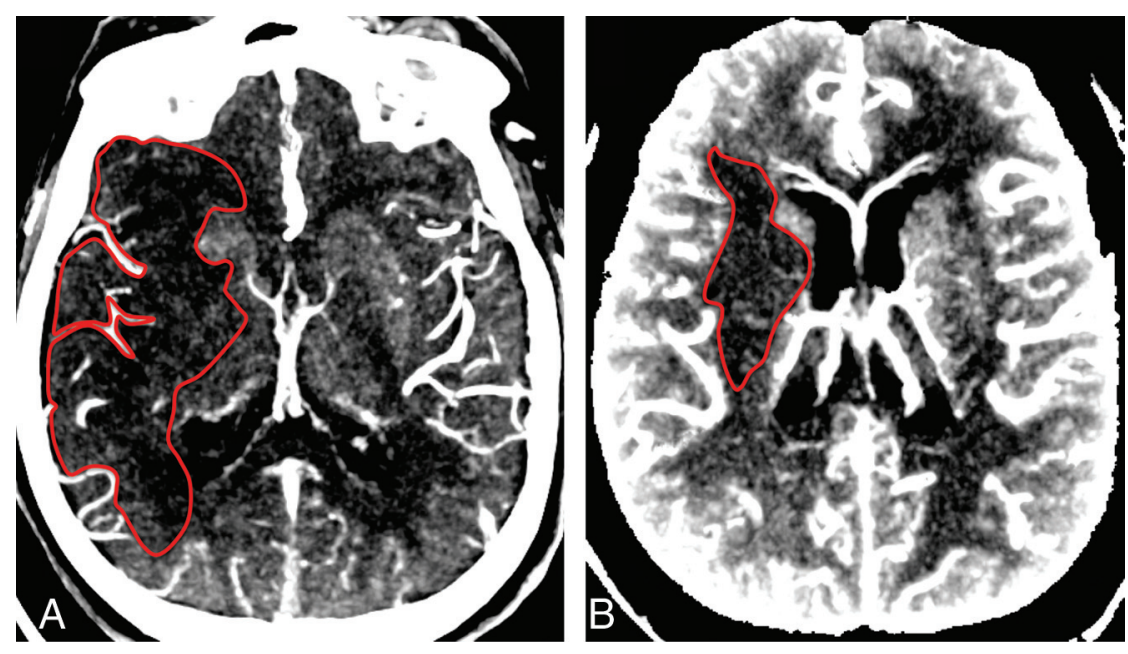

FIG 2. Comparison of conventional and dynamic CTA. A, MIP (10-mm) of conventional CTA source images. Volume of hypoattenuation (1 representative section outlined) is $90.5 \mathrm{~mL}$. B, Temporal MIPs $(10 \mathrm{~mm})$ of dynamic CTA source images reconstructed for the arteriovenous phase. Volume of hypoattenuation (1 representative section outlined) is $7.5 \mathrm{~mL}$. The follow-up infarct volume was $6.5 \mathrm{~mL}$.

of the hypoattenuation could be evaluated by comparison with the contralateral hemisphere. Figure 2 shows representative examples. The region volume was then calculated by using the OsiriX volume calculation tool. According to previous studies on the quantification of the region of hypoattenuation, ${ }^{13}$ window and level settings were adjusted by the reader to increase the contrast between normal and hypoattenuated brain tissue.

Grading of Collateral Vessels. All images were reconstructed with a section thickness of $20 \mathrm{~mm}$ to increase the visualization of vessel continuity. We assessed collateralization in conventional CT angiography and temporal MIPs of the arterial, arteriovenous, and venous phases. Collateral vessels were graded by using 3 previously described and evaluated scores (On-line Table 1). For relative collateral grading in dynamic CT angiography, we used contralateral arterial vessel status as a reference to prevent venous superimposition. Collaterals were assessed separately for each phase and each score.

In addition, we assessed CT angiographic datasets for an additional ICA occlusion and a visible early temporal branch before the occlusion because these have been shown to affect final infarct size. $^{22}$

\section{Follow-Up Image Analysis}

MR imaging or NECT acquired at least 1 day after the initial multiparametric CT was used to assess follow-up lesion volume. In MR imaging, lesions were assessed as volumes of relatively increased signal in the DWI sequence with a b-value of 1000 $\mathrm{s} / \mathrm{mm}^{2}$ and concomitant decreases in the apparent diffusion coefficient. On NECT, lesions were assessed as volumes of relative hypoattenuation. Volumetric analyses of either MR imaging or NECT datasets were performed by using OsiriX, Version 4.0, as described above, for regions of hypoattenuation analysis.

Image analysis was performed by 2 readers: 1 board-certified radiologist (W.H.S.) with 10 years of experience in neuroimaging, and 1 reader (S.E.B.) with 2 years of experience in acute stroke imaging. Quantitative measurements (eg, drawing of ROIs) were performed by the less experienced reader. In unclear cases, the more experienced reader was consulted. The readers were blinded to clinical data and follow-up imaging.

\section{Statistical Analysis}

We performed all statistical analyses by using SPSS (Version 22.0; IBM, Armonk, New York) and the open-source R statistical computing software, Version 3.0.3 (http://www.r-project.org).

All metric and normally distributed variables are reported as mean $\pm \mathrm{SD}$; non-normally distributed variables are presented as median with first-third quartiles. Categoric variables are presented as frequency and percentage.

Normal distribution was assessed by using histogram methods for the variable follow-up lesion volume. Square root transformation was performed for normalization of the dependent variable in regression analysis in case of non-normal distribution. Univariate linear regression analysis was used to test the association between predictors and follow-up lesion volume as outcome. The following variables were included as predictors: age, sex, time from symptom onset to initial imaging, time to follow-up imaging, additional ICA occlusion, visible early temporal branch, intravenous thrombolysis, and mechanical recanalization.

Multivariate linear regression was used for adjusted analyses of collateral scores and volume of hypoattenuation for follow-up lesion volume. Variables significantly associated with a favorable outcome $(P<.2)$ in the univariate regression were included in the multivariate models with the collateral scores or volume of hypoattenuation. The 4-point absolute and the 5-point relative scores were entered as ordinal variables. Volume of hypoattenuation and the 20-point relative score were entered as continuous variables. $P$ values $<.05$ were statistically significant. Residual plots (independent variable: regression standardized predicted value; dependent variable: regression standardized residual) and normal probability-probability plots of regression standardized residuals were generated to evaluate linear regression models.

Multivariate regression models were compared on the basis of the adjusted coefficients of determination (adjusted $R^{2}$ ) and the Bayesian information criterion (BIC). Differences in BIC of 2-6 were regarded as "positive"; $6-10$, as "strong"; and $>10$, as "very strong."

\section{RESULTS}

\section{Study Population}

Among our cohort of 1912 patients and after exclusion of noncomplete raw datasets, 165 patients showed complete M1 occlusion on conventional and dynamic CT angiography and were included in further analyses. Among these, we further excluded 22 patients due to missing follow-up imaging and 7 patients due to nondiagnostic quality of dynamic CTA. The remaining $136 \mathrm{pa}-$ tients constituted the study cohort. Figure 3 illustrates the detailed patient flow chart of the study cohort. 


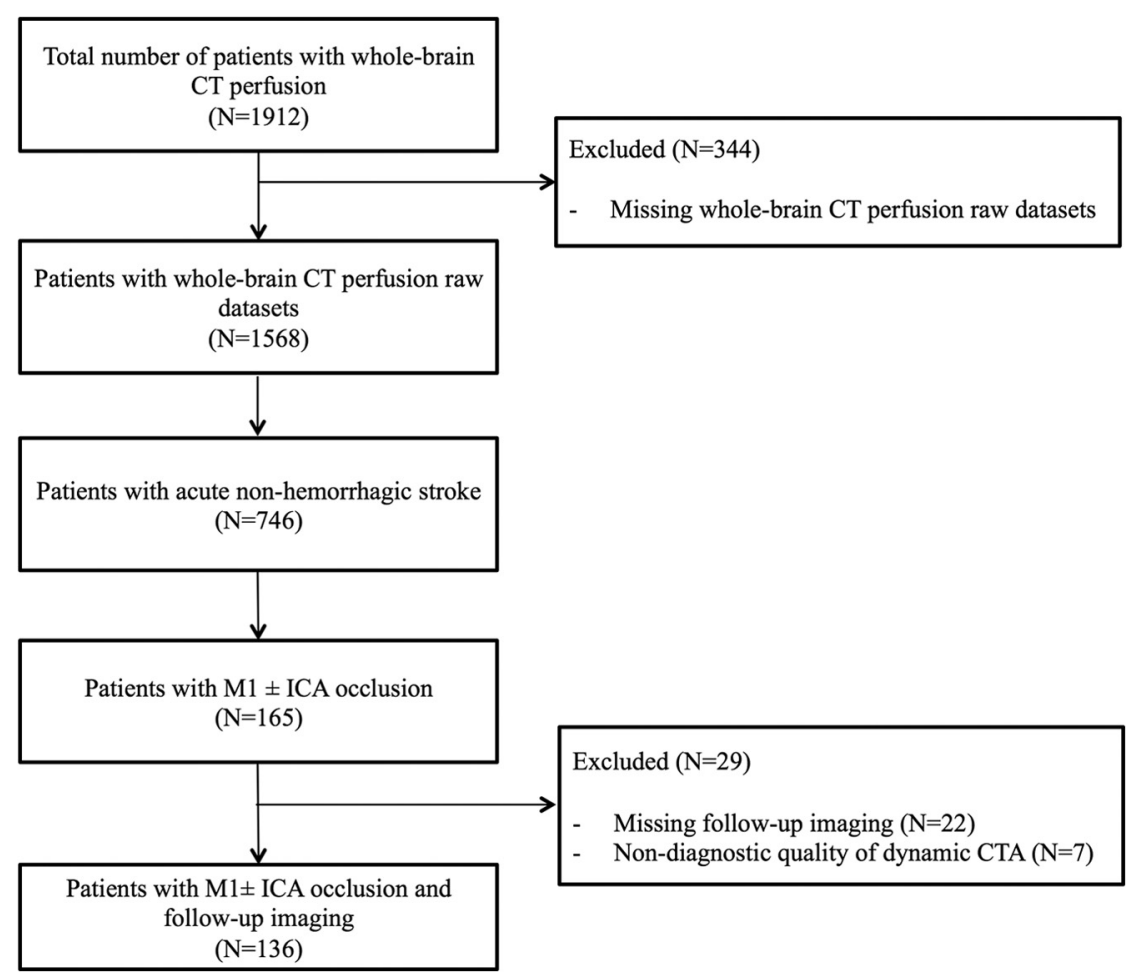

FIG 3. Inclusion and exclusion flow chart.

Table 1: Patient characteristics and univariate linear regression analysis to determine the effect of patient characteristics on follow-up lesion size

\begin{tabular}{|c|c|c|c|}
\hline & $\begin{array}{l}\text { All Patients } \\
(N=136)\end{array}$ & $\boldsymbol{\beta}$-Coefficient ${ }^{a}$ & $P$ Value $^{\mathrm{a}}$ \\
\hline Age $(y r)^{b}$ & $70.4 \pm 13.8$ & $\beta=-0.043$ & .235 \\
\hline Male sex (No.) (\%) & $56(41.2)$ & $\beta=1.434$ & .157 \\
\hline $\begin{array}{l}\text { Time from symptom onset to initial imaging }{ }^{c} \\
(\mathrm{~min})^{d}\end{array}$ & $139(89-227)$ & $\beta=-0.001$ & .897 \\
\hline Time to follow-up imaging (days) ${ }^{d}$ & $3(1-5)$ & $\beta=-0.095$ & .051 \\
\hline Additional ICA occlusion (No.) (\%) & $65(47.8)$ & $\beta=4.911$ & $<.001$ \\
\hline Visible early temporal branch (No.) (\%) & $28(20.6)$ & $\beta=-2.431$ & .048 \\
\hline IV thrombolysis (No.) (\%) & $90(66.2)$ & $\beta=-3.926$ & $<.001$ \\
\hline Mechanical recanalization (No.) (\%) & $72(52.9)$ & $\beta=-3.776$ & $<.001$ \\
\hline Follow-up lesion volume ${ }^{\mathrm{e}}(\mathrm{mL})^{\mathrm{d}}$ & $79(19-218)$ & N/A & N/A \\
\hline
\end{tabular}

Note:- N/A indicates not available.

${ }^{a}$ Results of univariate linear regression analysis with follow-up lesion volume (square-root-transformed) as a dependent variable. Positive $\beta$-values indicate an increase in follow-up lesion volume.

${ }^{\mathrm{b}}$ Mean.

${ }^{c}$ Documented for 70 patients.

${ }^{\mathrm{d}}$ Median (first-third quartile).

${ }^{e}$ As measured by DWI-MRI $(b=1000)$ or NECT.

\section{Baseline Characteristics}

The mean age was $70 \pm 14$ years (range, 28-97 years). Fifty-six $(41.2 \%)$ patients were male. Ninety patients $(66.2 \%)$ were treated with IV thrombolysis, and 72 patients (52.9\%) underwent mechanical recanalization. Among these, 57 patients (41.9\%) were treated with IV thrombolysis and mechanical recanalization. Median follow-up lesion volume was $79 \mathrm{~mL}$ (first-third quartiles = 19-218). Because follow-up lesion volume was not normally distributed, square root transformation was performed for this variable for further analysis. Patient characteristics and the results of univariate linear regression analyses for the square-root-transformed follow-up lesion volume are given in Table 1 .

Collateral grades were lowest on conventional CTA. In dynamic
CT angiography, collateral grades were highest in the arteriovenous phase and were slightly lower in the arterial phase than in the venous phase. The volume of hypoattenuation was highest in conventional CTA and decreased continuously from the arterial to the venous phase. The detailed results of collateral vessel grading systems are presented in On-line Table 2.

\section{Optimal Time-Phase for Collateral Assessment}

The models containing collateral grading assessed in the arteriovenous phase of dynamic CTA showed the best model fit (highest adjusted $R^{2}$, Table 2) in multivariate linear regression analyses for follow-up lesion volume controlling for the variables sex, additional ICA occlusion, time to follow-up imaging, early temporal branch, IV thrombolysis, and mechanical recanalization. This was seen independent of whether the models contained the volume of hypoattenuation or any collateral grading score.

For the model containing the volume of hypoattenuation, model fit (adjusted $R^{2}$ ) was 0.614 in the arteriovenous phase, compared with 0.483 in conventional CTA (BIC difference $=39.9$, indicating a very strong difference between the 2 models), 0.576 in the arterial phase (BIC difference $=15.8$, also indicating a very strong difference), and 0.586 in the venous phase (BIC difference $=$ 9.6, indicating a strong difference). Figure 2 presents examples comparing conventional and dynamic CT angiography in a typical patient. On-line Fig 1 illustrates the plots of the residuals of the multivariate regression models.

For the model containing the 4-point absolute score, BIC difference between the arteriovenous phase and the conventional CTA, arterial phase, and venous phase was 16.7 (very strong), 5.7 (positive), and 20.7 (very strong), respectively. For the model containing the 5-point relative score, the BIC difference between the arteriovenous phase and conventional CTA, arterial phase, and venous phase was 17.1 (very strong), 16.8 (very strong), and 26.5 (very strong), respectively. For the model containing the 20-point relative score, the BIC difference between the arteriovenous phase and conventional CTA, arterial phase, and venous phase was 12.0 (very strong), 15.6 (very strong), and 27.4 (very strong), respectively (Fig 4).

\section{Volume of Hypoattenuation versus Collateral Vessel Grading Scores}

In the multivariate linear regression analysis for follow-up lesion volume controlling for the variables sex, additional ICA occlu- 
Table 2: Model fit of multivariate linear regression for follow-up lesion volume ${ }^{a}$

\begin{tabular}{lcccc}
\hline & & \multicolumn{3}{c}{ Dynamic CT Angiography $\left(R^{2}\right)$} \\
\cline { 2 - 5 } & $\begin{array}{c}\text { Conventional } \\
\text { CTA }\left(R^{2}\right)\end{array}$ & $\begin{array}{c}\text { Arterial } \\
\text { Phase }\end{array}$ & $\begin{array}{c}\text { Arteriovenous } \\
\text { Phase }\end{array}$ & $\begin{array}{c}\text { Venous } \\
\text { Phase }\end{array}$ \\
\hline 4-Point absolute & 0.392 & 0.439 & 0.462 & 0.374 \\
5-Point relative & 0.348 & 0.349 & 0.441 & 0.321 \\
20-Point relative & 0.436 & 0.421 & 0.483 & 0.368 \\
Volume of hypoattenuation & 0.483 & 0.567 & 0.614 & 0.586 \\
\hline
\end{tabular}

Note:- $R^{2}$ indicates the adjusted coefficient of determination corrected for sex, time to follow-up imaging, additional ICA occlusion, visible early temporal branch, IV thrombolysis, and mechanical recanalization. It is an indicator of model fit; higher values indicate a better explanation of the variance of the outcome variable.

a Overall, dynamic CTA allows a better prediction of follow-up lesion volume than conventional CTA. Specifically, model fit was highest for reconstructions of dynamic CTA images within the arteriovenous phase. Models containing the volume of hypoattenuation performed better than models containing any of the collateral vessel grading scores.

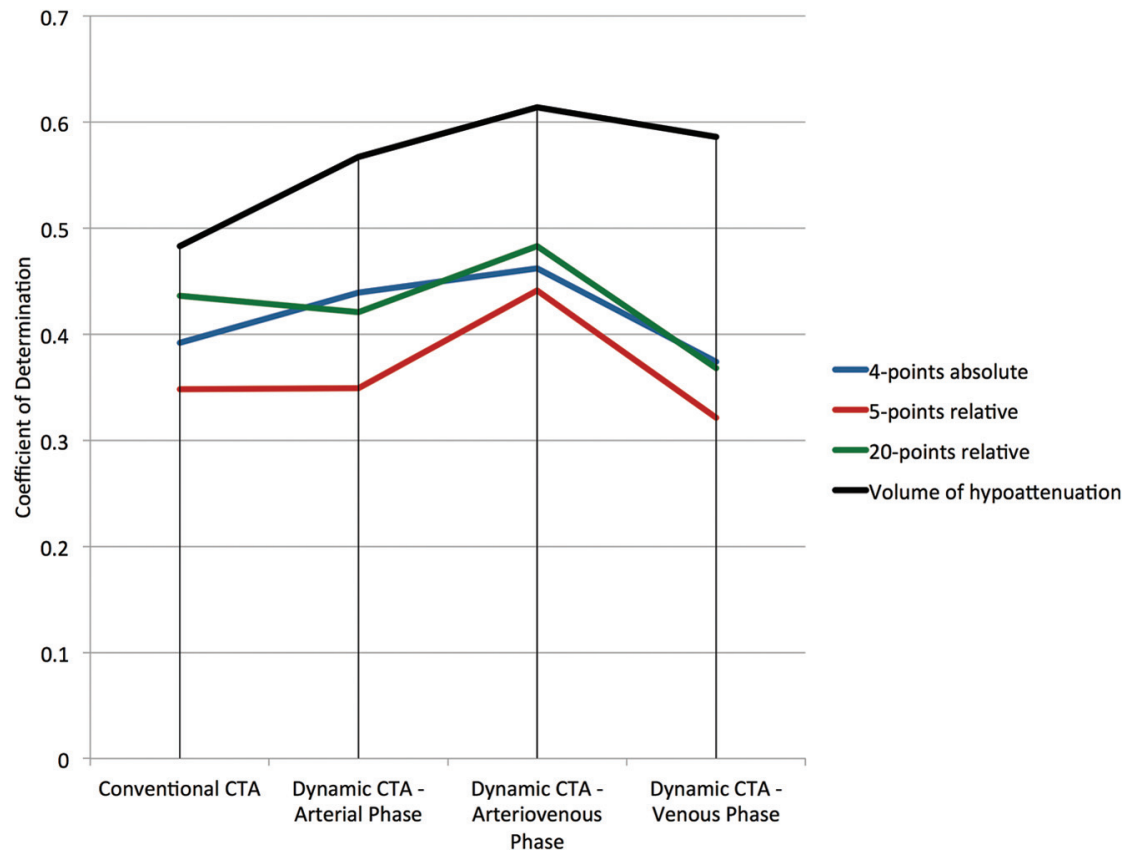

FIG 4. Comparison of collateral grading systems. The coefficient of determination is an indicator of model fit. Higher values indicate a better explanation of the variance of the outcome variable.

sion, time to follow-up imaging, early temporal branch, intravenous thrombolysis, and mechanical recanalization, the models containing a volume of hypoattenuation showed a better model fit (adjusted $R^{2}$ ) than models containing any of the collateral vessel grading scores (adjusted $R^{2}=0.483$ versus $0.392-0.436$ in conventional CTA, 0.567 versus $0.349-0.439$ in the arterial phase, 0.614 versus $0.441-0.483$ in the arteriovenous phase, 0.586 versus $0.321-0.374$ in the venous phase, Table 2). BIC analyses confirmed these results and showed a very strong preference (BIC difference $=>10$ ) for models containing the volume of hypoattenuation in conventional CTA and in all phases of dynamic CTA.

Among collateral vessel grading scores, the BIC favored the model containing the 20-point relative score in conventional CTA (BIC difference $=17.8$, indicating a very strong difference). In dynamic CTA, there was also a preference for the 20-point relative score in the arterial phase (BIC difference $=3.3$ ), in the arteriovenous phase $(\mathrm{BIC}$ difference $=13.2$, indicating a very strong difference), and in the venous phase (BIC difference $=6.5$, indicating a strong difference). Figure 5 shows 2 examples of the volume of hypoattenuation and collateral vessels in all 3 phases of dynamic CTA and the infarct on follow-up in a typical patient.

\section{DISCUSSION}

Our data demonstrate the volume of hypoattenuation to be a stronger predictor of the follow-up lesion volume than collateral vessel grading scores. Dynamic CTA, particularly if assessed in the arteriovenous phase, was superior to conventional CTA in predicting the follow-up infarction volume.

Our findings are in line with and extend those of prior reports showing that the degree of collateralization is an independent predictor of tissue outcome. ${ }^{4,5,23}$ Most of these studies were performed by using conventional CTA for collateral assessment. So far, no consensus has been reached on how to standardize collateral assessment. Different approaches included the quantification of the volume of hypoattenuation in CTA source images $^{12,13}$ and the use of different collateral vessel grading scores. ${ }^{10}$

In our study, we demonstrate the volume of hypoattenuation to be a stronger predictor of tissue outcome than collateral vessel grading scores in both conventional and dynamic angiography. While collateral vessels correspond to the macrocirculation within the ischemic bed, attenuation values correlate with parenchymal contrast material arrival and may therefore be considered an indicator of effectiveness of collateral blood supply. ${ }^{24}$ This effectiveness may depend on not only the status of the macrocirculation but also other factors such as cerebral perfusion pressure ${ }^{1}$ and may therefore be a better prognostic marker.

Another crucial aspect in collateral blood flow imaging by using conventional CT angiography is the time point of image acquisition. Triggered protocols that cover the phase of peak arterial enhancement have already found their way into clinical routine because they reliably detect arterial occlusions, stenoses, and aneurysms. ${ }^{13,17}$ However, image acquisition at an early arterial phase can fail to capture delayed collateral contrast material arrival. ${ }^{17}$ Using delay-insensitive dynamic CTA, Smit et al ${ }^{17}$ could show that the presence of a delayed collateral enhancement may still be an important predictor of a good outcome. While important information has been added to the previously described value of conventional CT angiography, ${ }^{25}$ the optimal time point for collateral evaluation has not been assessed. Other studies demonstrated that the application of different CTA acquisition protocols has a substantial effect on the correlation with concur- 


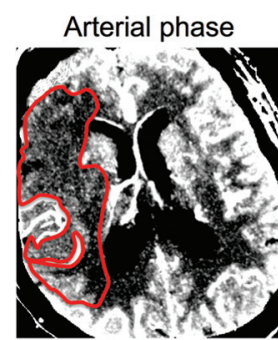

Lesion: $152.13 \mathrm{ml}$

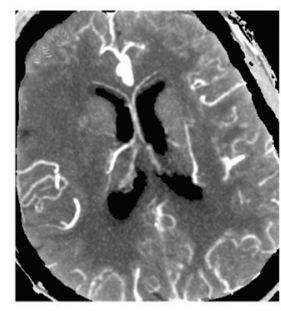

Collateral grades:

A $\quad 4,2,1$

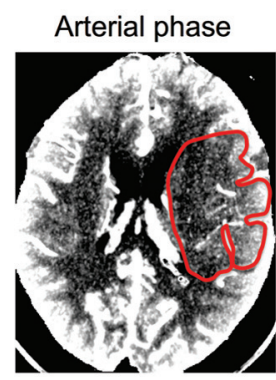

Lesion: $48.82 \mathrm{ml}$

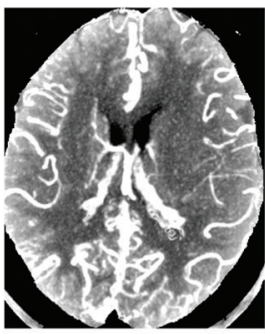

Collateral grades:

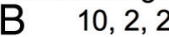

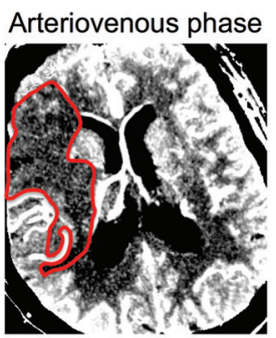

Lesion: $99.20 \mathrm{ml}$

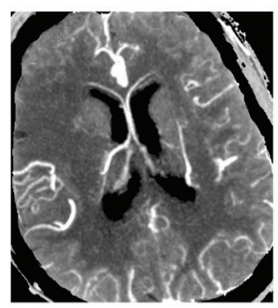

Collateral grades:

$7,2,1$

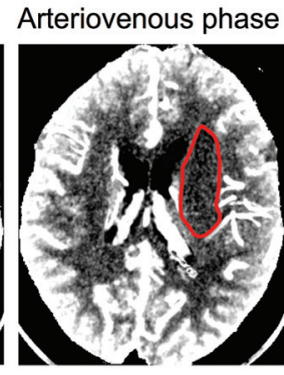

Lesion: $28.49 \mathrm{ml}$

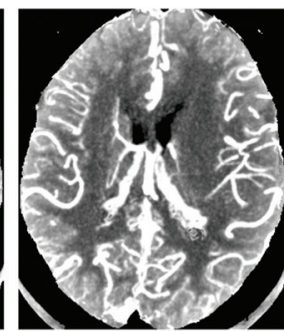

Collateral grades:

$16,4,3$

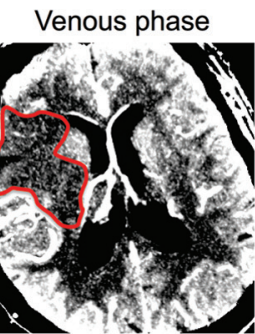

Lesion: $91.06 \mathrm{ml}$

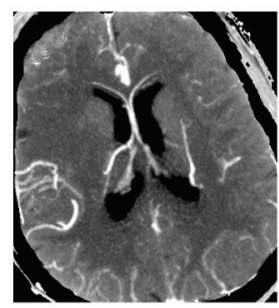

Collateral grades:

$6,2,1$

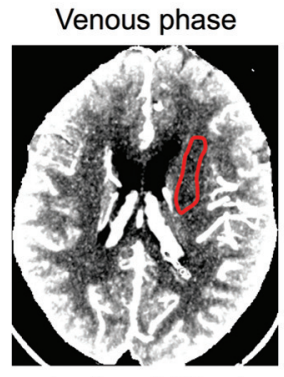

Lesion: $9.96 \mathrm{ml}$

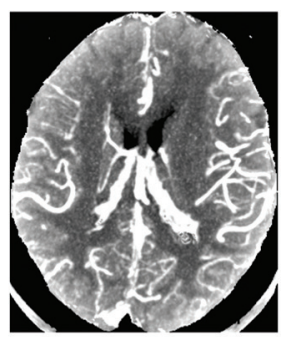

Collateral grades:

$18,5,3$

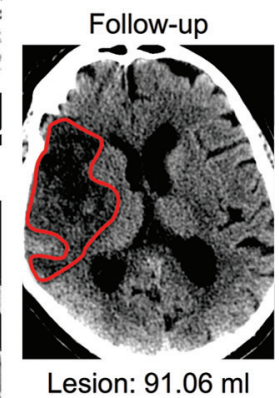

might fail to capture the full extent of collateral enhancement. ${ }^{17}$

Furthermore, our results indicate that collateral assessment in dynamic CTA during the venous phase has a worse prognostic value than assessment during an earlier phase. Two possible reasons for a worse prognostic performance of the late venous phase compared with the earlier arteriovenous phase may be an overestimation of collateral blood flow due to concurrent enhancement of venous vessels and an important role of collateral filling time, which cannot be accounted for in the late venous phase. The superiority of the arteriovenous phase may also have important implications for standardization of collateral blood flow assessment by using conventional CT angiography because it might not be necessary to perform image acquisition during a delayed venous phase. Instead, the arteriovenous phase might allow an optimal assessment of occlusions and of collateral blood flow.

Our data should be interpreted in the context of the study design. As a retrospective study, there is a potential for bias in patient selection. The use of a large cohort of consecutive patients and standardized protocols for patients with suspected stroke, however, helped to reduce these confounding factors. Furthermore, although patients with mechanical recanalization and treatment by intravenous rtPA were included, the state of recanalization between initial CT and follow-up imaging was not assessed. Lack of recanalization leads to larger volumes of infarction and reduces the correlation between initial and follow-up lesion volumes. ${ }^{14}$ Thus, different rates of recanalization might alter the correlation between the collateral status at different time-phases and the follow-up lesion volume. Finally, assessment was performed in consensus readings. The purpose of this study was rently acquired $\mathrm{CT}$ perfusion ${ }^{26}$ and MR diffusion-weighted images. ${ }^{13}$

Independent of the approach chosen to evaluate the collateralization status, our results suggest an optimal prognostic value of collateralization when assessed during the arteriovenous phase. Notably, the prognostic value was lowest in conventional CT angiography, which is acquired in a caudocranial direction with a 2-second delay after bolus triggering in the aortic arch (cutoff value $=100 \mathrm{HU}$ ) at our institution. This finding is in line with those in previous studies showing that early scan acquisition

not to test interobserver agreement but to compare the predictive value of different strategies at different time-phases.

\section{CONCLUSIONS}

We demonstrated that the type of collateral blood flow assessment is an important cornerstone for estimating the final infarct volume. Among all strategies tested, the use of dynamic CTA within the arteriovenous phase, including the quantification of the volume of hypoattenuation, is the best-suited technique to assess collateralization. This technique may help to reliably assess the 
collateralization status and to make a first prognostic statement in the acute stroke situation.

Disclosures: Maximilian F. Reiser—UNRELATED: Grants/Grants Pending: 1) German Research Society (Deutsche Forschungsgemeinschaft) "Cluster of Excellence"; 2 ) Euro-Biolmaging;* 3) German National Cohort; ${ }^{*}$ 4) Munich Cluster of Excellence M4 Imaging;* 5) Federal Ministry of Education and Research German Center for Lung Diseases; ${ }^{*}$ and 6) Federal Ministry of Education and Research German Center for Cardiovascular Diseases, ${ }^{*}$ Comments: 1) November 2006-2016 Principal Investigator, Munich Center of Advanced Photonics, Ludwig Maximilians University of Munich, Technical University of Munich, Germany; December 20, 2010-2016 Principal Site Investigator Euro-Biolmaging: European Commission-SP4 Capacities-FP7-Infrastructures-2010-1 (GA 262023). Ludwig Maximilians University of Munich, Germany; 3) 20112016 Co-Principal Investigator, German National Cohort, Imaging Working GroupWhole Body MRI Phenotyping, Federal Ministry of Education and Research/ Helmholtz Society, Germany; 4) 2011-2016 Co-Principal Investigator: ImagingPersonalized Medicine, a new dimension of drug development; 5) 2010-2015 Principal Investigator; and 6) 2010-2015 Principal Investigator. Birgit Ertl-Wagner-RELATED: Grant: German Research Council (past)*; UNRELATED: Board Membership: Philips Healthcare (current), Bracco (current), Springer Medical Publishing (current); Consultancy: Munich Medical International (current), Philips Healthcare (current); Grants/ Grants Pending: Eli Lily (current), ${ }^{*}$ Genentech (current), ${ }^{*}$ Guerbet (current), ${ }^{*}$ Merck Serono (current), ${ }^{*}$ Novartis (current); ${ }^{*}$ Payment for Lectures (including service on Speakers Bureaus): Siemens (ongoing), Bayer-Schering (past); Payment for Manuscript Preparation: Siemens (past); Springer Medical Publishing (ongoing), Thieme Medical Publisher (ongoing), Bracco (ongoing); Royalties: Springer Medical Publishing (ongoing), Thieme Medical Publisher (ongoing); Payment for Development of Educational Presentations: Siemens (past), Bracco (ongoing), Springer (ongoing), Thieme (ongoing); Stock/Stock Options: Siemens (stock owned by spouse, ongoing); Travel/ Accommodations/Meeting Expenses Unrelated to Activities Listed: Siemens (ongoing). *Money paid to the institution.

\section{REFERENCES}

1. Liebeskind DS. Collateral circulation. Stroke 2003;34:2279-84

2. Tariq N, Khatri R. Leptomeningeal collaterals in acute ischemic stroke. J Vasc Interv Neurol 2008;1:91-95

3. Tan JC, Dillon WP, Liu S, et al. Systematic comparison of perfusion-CT and CT-angiography in acute stroke patients. Ann Neurol 2007;61:533-43

4. Miteff F, Levi CR, Bateman GA, et al. The independent predictive utility of computed tomography angiographic collateral status in acute ischaemic stroke. Brain 2009;132:2231-38

5. Calleja AI, Cortijo E, Garcia-Bermejo P, et al. Collateral circulation on perfusion-computed tomography-source images predicts the response to stroke intravenous thrombolysis. Eur J Neurol 2013;20:795-802

6. Jung S, Gilgen M, Slotboom J, et al. Factors that determine penumbral tissue loss in acute ischaemic stroke. Brain 2013;136:3554-60

7. Lima FO, Furie KL, Silva GS, et al. The pattern of leptomeningeal collaterals on CT angiography is a strong predictor of long-term functional outcome in stroke patients with large vessel intracranial occlusion. Stroke 2010;41:2316-22

8. Maas MB, Lev MH, Ay H, et al. Collateral vessels on CT angiography predict outcome in acute ischemic stroke. Stroke 2009;40:3001-05

9. Campbell BC, Christensen S, Tress BM, et al. Failure of collateral blood flow is associated with infarct growth in ischemic stroke. J Cerebr Blood Flow Metab 2013;33:1168-72

10. McVerry F, Liebeskind DS, Muir KW. Systematic review of methods for assessing leptomeningeal collateral flow. AJNR Am J Neuroradiol 2012;33:576-82

11. Menon BK, Smith EE, Modi J, et al. Regional leptomeningeal score on CT angiography predicts clinical and imaging outcomes in patients with acute anterior circulation occlusions. AJNR Am J Neuroradiol 2011;32:1640-45

12. Schaefer PW, Yoo AJ, Bell D, et al. CT angiography-source image hypoattenuation predicts clinical outcome in posterior circulation strokes treated with intra-arterial therapy. Stroke 2008;39:3107-09

13. Pulli B, Schaefer PW, Hakimelahi R, et al. Acute ischemic stroke: infarct core estimation on CT angiography source images depends on CT angiography protocol. Radiology 2012;262:593-604

14. Lev MH, Segal AZ, Farkas J, et al. Utility of perfusion-weighted CT imaging in acute middle cerebral artery stroke treated with intraarterial thrombolysis: prediction of final infarct volume and clinical outcome. Stroke 2001;32:2021-28

15. Schramm P, Schellinger PD, Fiebach JB, et al. Comparison of CT and CT angiography source images with diffusion-weighted imaging in patients with acute stroke within 6 hours after onset. Stroke 2002;33:2426-32

16. Dzialowski I, Weber J, Doerfler A, et al. Brain tissue water uptake after middle cerebral artery occlusion assessed with CT. J Neuroimaging 2004;14:42-48

17. Smit EJ, Vonken EJ, van Seeters T, et al. Timing-invariant imaging of collateral vessels in acute ischemic stroke. Stroke 2013;44:2194-99

18. Frölich AM, Wolff SL, Psychogios MN, et al. Time-resolved assessment of collateral flow using 4D CT angiography in large-vessel occlusion stroke. Eur Radiol 2014;24:390-96

19. Bivard A, Levi C, Spratt N, et al. Perfusion CT in acute stroke: a comprehensive analysis of infarct and penumbra. Radiology 2013;267:543-50

20. Smit EJ, Vonken EJ, van der Schaaf IC, et al. Timing-invariant reconstruction for deriving high-quality CT angiographic data from cerebral CT perfusion data. Radiology 2012;263:216-25

21. Thierfelder KM, Sommer WH, Baumann AB, et al. Whole-brain CT perfusion: reliability and reproducibility of volumetric perfusion deficit assessment in patients with acute ischemic stroke. Neuroradiology 2013;55:827-35

22. Menon BK, Bal S, Modi J, et al. Anterior temporal artery sign in CT angiography predicts reduced fatal brain edema and mortality in acute M1 middle cerebral artery occlusions. J Neuroimaging 2012;22:145-48

23. Kim JJ, Fischbein NJ, Lu Y, et al. Regional angiographic grading system for collateral flow: correlation with cerebral infarction in patients with middle cerebral artery occlusion. Stroke 2004;35: $1340-44$

24. Shuaib A, Butcher K, Mohammad AA, et al. Collateral blood vessels in acute ischaemic stroke: a potential therapeutic target. Lancet Neurol 2011;10:909-21

25. Souza LC, Yoo AJ, Chaudhry ZA, et al. Malignant CTA collateral profile is highly specific for large admission DWI infarct core and poor outcome in acute stroke. AJNR Am J Neuroradiol 2012;33: 1331-36

26. Sharma M, Fox AJ, Symons S, et al. CT angiographic source images: flow- or volume-weighted? AJNR Am J Neuroradiol 2011; $32: 359-64$ 